\title{
Real-time needle detection and tracking using a visually servoed 3D ultrasound probe
}

\author{
Pierre Chatelain, Alexandre Krupa and Maud Marchal
}

\begin{abstract}
In this paper, we present a method to localize and track manually inserted needles in real-time using a threedimensional ultrasound probe mounted on a robotized arm. The system tracks the needle using online image processing. We first propose a new algorithm capable of robustly detecting a needle from the moment it is inserted, without any a priori information on the insertion direction. By combining the random sample consensus (RANSAC) algorithm with Kalman filtering in closed loop, we achieve robust real-time tracking of the needle. In addition, we propose a control scheme to automatically guide the ultrasonic probe in order to keep the needle within the field of view, while aligning its axis with the ultrasound beam. This method will ease the insertion of the needle by the operator, and allow the development of autonomous needle insertion by medical robots.
\end{abstract}

\section{INTRODUCTION}

In minimally invasive surgical procedures such as biopsies, a thin flexible needle has to be inserted into the patient's body, and guided accurately toward a soft tissue target. Recent studies have shown the potential of ultrasound (US) imaging for guiding minimally invasive surgical interventions such as needle insertion when direct vision is not possible [1]. However, US images have a high level of noise and can contain artifacts such as shadowing or reverberation, often making their interpretation challenging. In addition, freehand manipulation of the US probe can lack the critical level of control and accuracy required for needle insertion procedures. Therefore robust image analysis techniques can greatly help the operator during an insertion procedure, by enhancing the needle visibility or automatically displaying its location on the US image [2].

In addition to the enhancement of tool visibility, US imaging is used for automatic tool guidance [3], [4]. For these tasks the US probe is maintained still, while the surgical tool is controlled by a robotic system. Novotny et al. [5] guide a surgical instrument to a tracked soft tissue target by 3D ultrasonic visual servoing. For other applications such as diagnosis or motion compensation, the US probe can also be controlled by a robotized system. Salcudean et al. [6] use visual servoing to track the carotid artery, detected in 2D ultrasound images with active contours. Mebarki et al. [7] achieve visual servoing of a 2D US probe based on image moments. Nadeau and Krupa [8] track and compensate organ motion using intensity-based visual servoing.

P. Chatelain is with Ecole Normale Supérieure de Cachan, Antenne de Bretagne, France. A. Krupa is with INRIA Rennes - Bretagne Atlantique, IRISA, France. M. Marchal is with INSA, IRISA, INRIA Rennes - Bretagne Atlantique, France. pierre.chatelaineens-cachan.fr alexandre.krupa@inria.fr maud.marchaleirisa.fr
In this paper, we propose a new configuration, using visual servoing to track a manually inserted biopsy needle with an ultrasound probe mounted on a robotic arm. Our aim is to assist the operator by keeping the needle tip visible and centered in the image, and the needle axis aligned with the longitudinal axis of the probe, thus improving its visibility.

This servoing task requires a robust real-time detection of the needle. Most of the existing needle localization methods in the literature are based on parallel projections, which are designed to find imperfect instances of parameterized shapes by optimizing the integral of the image along parallel curves. Hong et al. [3] use the Hough transform (HT) to detect straight needles in two-dimensional US images. Aboofazeli et al. [9] adapt this method to the detection of mildly curved needles in a 3D volume, by first projecting the volume onto 2D planes by a ray casting process. Several variants of the Hough transform allow to reduce the computational time, using coarse-fine strategies [10] or randomization [11]. Barva et al. [12] use the parallel integral projection (PIP) to localize straight needles in 3D volumes. Uherčik et al. [13] develop a multi-resolution scheme (MR-PIP) to speed-up the computation. Novotny et al. [5] optimize this algorithm for an implementation on graphics processing unit (GPU) to achieve real-time detection. Although the Hough transform was first designed for the detection of straight lines, it is applicable to any parameterized curve. Neshat and Patel [14] represent a curved needle by a Bézier polynomial, and optimize its parameters using the Radon transform (equivalent to PIP). The algorithm is also implemented on GPU for real-time detection.

However, projection-based methods have a relatively high computational time on large volumes, even with a parallel implementation, and lack of robustness on cluttered background. As a more robust alternate method, Uherčik et al. [15] propose to use the random sample consensus (RANSAC) algorithm to detect polynomial curves in 3D volumes. The result is then optimized locally using linear regression, to compensate for the relatively poor accuracy of the RANSAC algorithm. Zhao et al. [16] improve the stability of the detection in a sequence of volumes by filtering the results using a Kalman filter.

In this paper, we propose to take advantage of the predictive power of the Kalman filter to speed-up the detection algorithm. This speed-up is achieved in part by reducing the search area based on the predicted position, and in part by discarding unlikely configurations during the RANSAC algorithm. In order to detect the needle from the beginning of its insertion, we also propose to use the image differences 
and look for a local displacement of high intensity voxels. Indeed, the RANSAC algorithm and other line-detection methods based on the intensity presented in the literature lack of robustness in the presence of other bright structures, and are rather slow when applied to large volumes. Thus these methods usually require a manual initialization or the selection of a region of interest in order to achieve robust real-time detection [17]. Our method, using the image differences to detect an initial motion, allows to detect a manual insertion in an arbitrary direction in real-time, without any prior information. The tracking algorithm then only starts when the needle has been inserted with a sufficient length.

We describe our detection method in section II, and our servoing system in section III. In section IV we present the localization and tracking results obtained during experiments on phantoms.

\section{Detection ALGORITHM}

The needle typically appears in the $3 \mathrm{D}$ volume as a thin, possibly curved line of high intensity voxels. However, artifacts or other objects can also appear with high intensity, making the static detection of the needle a difficult task. We propose therefore to use the motion information induced by the insertion of the needle. A simple way to detect motion is to compute the difference of intensity between consecutive volumes [2], [18]. However, this method cannot be used during the tracking in our setup, as the ultrasound probe is not static.

\section{A. Method overview}

The method consists first in detecting changes of intensity in consecutive frames to detect possible location of a moving needle (section II-B). Once the needle is detected and visible with a certain length in the volume, we localize it with a RANSAC-based method (section II-D), improved using the predictive information provided by a Kalman filter (section II-C).

\section{B. Insertion detection}

The ultrasound probe provides a sequence of $3 \mathrm{D}$ volumes. The intensity of each voxel of the volume is defined by $I_{t}$ : $\mathcal{V} \longrightarrow \mathbb{R}$, where $\mathcal{V} \subset \mathbb{R}^{3}$ is the set of voxels belonging to the volume. At each time $t$, we define the volume difference as the absolute difference $\Delta I_{t}=\left|I_{t}-I_{t-1}\right|$. A set $\widetilde{\mathcal{X}}_{t}$ of candidate voxels $\mathbf{v}$ is obtained by thresholding the volume difference, in order to detect important intensity changes:

$$
\widetilde{\mathcal{X}_{t}}=\left\{\mathbf{v} \in \mathcal{V}: \Delta I_{t}(\mathbf{v}) \geq T_{t}\right\}
$$

where the threshold $T_{t}$ is chosen so that the size $\left|\widetilde{\mathcal{X}_{t}}\right|$ of the candidate set is fixed.

We then use the variance of the candidate set to distinguish between a localized motion due to the insertion of the needle, and a global motion of the tissues. The motion of the needle indeed creates localized high intensity changes, and thus the variance of the candidate set will be lower when the needle is moving. We only proceed to the next steps if the variance is lower than a threshold $\tau$, and otherwise we wait for the next volume. In order to make the variance criterion efficient, we empirically chose a size of candidate voxels $\left|\widetilde{\mathcal{X}_{t}}\right|$ that maximized the difference of variance between a motionless volume and a volume containing the moving needle.

If the variance is lower than $\tau$, we compute the geometric median of the candidate set to localize the entry point:

$$
\operatorname{Med}\left(\widetilde{\mathcal{X}_{t}}\right)=\arg \min _{\mathbf{y} \in \mathbb{R}^{3}} \sum_{\mathbf{x} \in \widetilde{\mathcal{X}}_{t}}\|\mathbf{x}-\mathbf{y}\|
$$

The geometric median is a robust estimator of data center location, and can be evaluated efficiently using Weiszfeld's algorithm [19], a form of iteratively re-weighted least squares. We use the geometric mean to initialize the Weiszfeld's algorithm.

The median is then projected onto the closest face of the volume boundary to obtain a candidate entry point $\mathbf{E}_{t}$. In order to improve the robustness of the detection, we consider the entry point as detected only if the distance $\left\|\mathbf{E}_{t}-\operatorname{Med}\left(\widetilde{\mathcal{X}_{t}}\right)\right\|$ between the entry point and the geometric median is smaller than a threshold $\delta$.

Once a valid entry point has been detected, we start the tracking algorithm.

\section{Kalman filtering}

During the tracking phase, the needle is modeled by a polynomial curve $\mathcal{C}_{t}$ of order $n$ (typically 2 for a straight needle or 3 for a flexible needle), represented by $n$ control points $\mathbf{p}_{1, t}\left(x_{1, t}, y_{1, t}, z_{1, t}\right), \ldots, \mathbf{p}_{n, t}\left(x_{n, t}, y_{n, t}, z_{n, t}\right)$. A Kalman filter is used to filter the localization results, and to predict the future position of the needle. The model state consists in the position and velocity of the $n$ control points:

$$
\mathbf{X}_{t}=\left(\mathbf{p}_{1}, \ldots, \mathbf{p}_{n}, \dot{\mathbf{p}}_{1}, \ldots, \dot{\mathbf{p}}_{n}\right)
$$

Noting $\mathbf{Z}_{\mathbf{t}}$ the measurement returned by the localization algorithm (section II-D), the dynamics of the system are modeled by the following linear equation:

$$
\begin{aligned}
\mathbf{X}_{t} & =\mathbf{A X}_{t-1}+\mathbf{u}_{t} \\
\mathbf{Z}_{t} & =\mathbf{C X}_{t}+\mathbf{v}_{t}
\end{aligned}
$$

with Gaussian noise terms

$$
\begin{aligned}
& \mathbf{u}_{t} \sim \mathcal{N}(\mathbf{0}, \mathbf{Q}) \\
& \mathbf{v}_{t} \sim \mathcal{N}(\mathbf{0}, \mathbf{R})
\end{aligned}
$$

where $\mathbf{Q}$ and $\mathbf{R}$ correspond respectively to the covariance matrix related to the model noise and the covariance matrix of the measurement noise.

When the measurement rate is high enough, the speed can be assumed constant, small accelerations being modeled by the state noise. Under this assumption, the transition matrix can be written as follows:

$$
\mathbf{A}=\left[\begin{array}{cc}
\mathbf{I}_{3 n} & \delta t \mathbf{I}_{3 n} \\
0 & \mathbf{I}_{3 n}
\end{array}\right]
$$

where $\delta t$ is the time step between two observations, and $\mathbf{I}_{3 n}$ corresponds to the $3 n \times 3 n$ identity matrix. The observation matrix is given by:

$$
\mathbf{C}=\left[\begin{array}{ll}
\mathbf{I}_{3 n} & 0
\end{array}\right]
$$


Then the Kalman filter equations can be split in two time update equations:

$$
\begin{aligned}
& \hat{\mathbf{X}}_{t}^{-}=\mathbf{A} \hat{\mathbf{X}}_{t-1} \\
& \mathbf{P}_{t}^{-}=\mathbf{A} \mathbf{P}_{t-1} \mathbf{A}^{T}+\mathbf{Q}
\end{aligned}
$$

and three measurement update equations:

$$
\begin{aligned}
\mathbf{K}_{t} & =\mathbf{P}_{t}^{-} \mathbf{C}^{T}\left(\mathbf{C P}_{t}^{-} \mathbf{C}^{T}+\mathbf{R}\right)^{-1} \\
\hat{\mathbf{X}}_{t} & =\hat{\mathbf{X}}_{t}^{-}+\mathbf{K}_{t}\left(\mathbf{Z}_{t}-\mathbf{C} \hat{\mathbf{X}}_{t}^{-}\right) \\
\mathbf{P}_{t} & =\left(\mathbf{I}-\mathbf{K}_{t} \mathbf{C}\right) \mathbf{P}_{t}^{-}
\end{aligned}
$$

In this study we do not have any a priori information on the needle motion, as it is inserted manually. In the case of robot-driven needle insertion, an extended Kalman filter [20] could be used to take into account the input needle motions.

\section{Needle localization}

In addition to filtering measurement noise, as it is done in [16], the Kalman filter can be used as a predictor to reduce the search area. We thus define a volume of interest (VOI) $\mathcal{V}_{t}^{-} \in \mathcal{V}$ around the position predicted by the Kalman filter, and restrict the localization algorithm to this VOI.

During the tracking phase, using the volume difference is not reliable anymore, as the US probe is moving. Therefore we select the candidate voxels within the VOI by thresholding directly the intensity:

$$
\mathcal{X}_{t}=\left\{\mathbf{v} \in \mathcal{V}_{t}^{-}: I_{t}(\mathbf{v}) \geq T_{t}\right\}
$$

where the threshold $T_{t}$ is designed as previously (equation (1)).

Then a RANSAC algorithm is used to fit a polynomial curve to the candidate set, based on the method proposed by Uherčik et al. [15]. The curve $\mathcal{C}_{t}$ is parameterized by a matrix $\mathbf{H} \in \mathbb{R}^{3 \times n}$ such that:

$$
\mathcal{C}_{t}(a, \mathbf{H})=\mathbf{H}\left[\begin{array}{llll}
1 & a & \ldots & a^{n-1}
\end{array}\right]^{T}
$$

where $a \in \mathbb{R}$ is the curvilinear coordinate.

The model is associated to a cost function $f(\mathbf{v}, \mathbf{H})$ used to classify the voxels $\mathbf{v} \in \mathcal{V}_{t}^{-}$as belonging to the needle or to the background. Uherčik et al. [17] propose two different cost functions: a simple function based on the distance to the surgical instrument axis, and a more complete one modeling the intensity distributions of the instrument and the background. Although using a model of the intensity distributions yields a better accuracy, it requires a significantly higher computational time, and the distributions have to be previously learned from a training set. Thus we use in this study the distance to the needle axis as cost function:

$$
f(\mathbf{v}, \mathbf{H})=d(\mathbf{v}, \mathbf{H})
$$

When the order of the polynomial curve $n>2$, we use an approximation of the point-to-curve distance, using the principal direction vector $\mathbf{k}_{0}$ of the needle that we determine by fitting a straight line to the control points $\mathbf{p}_{i, t}$ (see Fig. 1):

$$
\hat{d}(\mathbf{v}, \mathbf{H})=\left\|\mathbf{v}-\mathcal{C}_{t}(\hat{a}, \mathbf{H})\right\|
$$

where $\hat{a}=\frac{\left(\mathbf{v}-\mathbf{p}_{1, t}\right) \cdot \mathbf{k}_{0}}{\left\|\mathbf{k}_{0}\right\|}$ is the approximate curvilinear coordinate of the projection of the voxel $\mathbf{v}$ onto the curve $\mathcal{C}_{t}$.

Then the set of inliers for a model $\mathbf{H}$ is defined as:

$$
\mathcal{X}_{t}^{\text {in }}(\mathbf{H})=\left\{\mathbf{v} \in \mathcal{X}_{t}: f(\mathbf{v}, \mathbf{H})<f_{0}\right\}
$$

where $f_{0}$ is a fix threshold.

The RANSAC algorithm optimizes the model $\mathbf{H}$ by repeating the following steps:

1) sample $n$ random candidates uniformly from $\mathcal{X}_{t}$;

2) evaluate the model $\mathbf{H}$ fitting this sample set;

3) estimate the set of inliers $\mathcal{X}_{t}^{\text {in }}(\mathbf{H})$. and returning the model yielding the largest inlier set $\hat{\mathcal{X}}_{t}^{\text {in }}$.

Given a failure threshold $\epsilon$, and the probability $p_{\text {in }}$ to select an inlier, the required number of iterations can be obtained as:

$$
T_{\text {iter }}=\frac{\log \epsilon}{\log \left(1-p_{\text {in }}^{n}\right)}
$$

Bounding $p_{\text {in }}$ by the largest proportion of inliers found, we obtain the stop criterion:

$$
\hat{T}_{\text {iter }}=\frac{\log \epsilon}{\log \left(1-\left(\left|\mathcal{X}_{t}^{\text {in }}\right| /\left|\mathcal{X}_{t}\right|\right)^{n}\right)}
$$

To avoid the computation of the cost function for unlikely configurations, Uherčik et al. use a constrained version of the RANSAC algorithm, as they do not proceed to the steps 2) and 3 ) for the models with excessively high curvature [17]. Here we propose in addition to use the prediction provided by the Kalman filter to discard incoherent needle configurations. This is done by computing the angle $\alpha$ between the principal directions $\mathbf{k}_{0}$ of the candidate model and $\mathbf{k}_{0}^{-}$of the predicted model:

$$
\alpha=\operatorname{acos}\left(\frac{\mathbf{k}_{0} \cdot \mathbf{k}_{0}^{-}}{\left\|\mathbf{k}_{0}\right\| \times\left\|\mathbf{k}_{0}^{-}\right\|}\right)
$$

and discarding models with $\|\alpha\|>\alpha_{0}$, where $\alpha_{0}$ is a predetermined threshold. The main advantages of introducing this new criterion are (i) a faster convergence, due to a reduced search space, and (ii) an improved robustness when the needle is close to other high-intensity objects.

The RANSAC algorithm gives a robust estimate of the model, but has poor accuracy, because it computes the parameters only from a minimal sample of $n$ points. Uherčik et al. perform a local optimization of the parameters using a derivative-free Nelder-Mead downhill simplex method [21], [22]. For the case $n=2$ we prefer to use the closedform solution of least squares regression to fit a line on the set of inliers $\hat{\mathcal{X}}_{t}^{\text {in }}$. The combination of RANSAC and local optimization ensures a robust and accurate estimation.

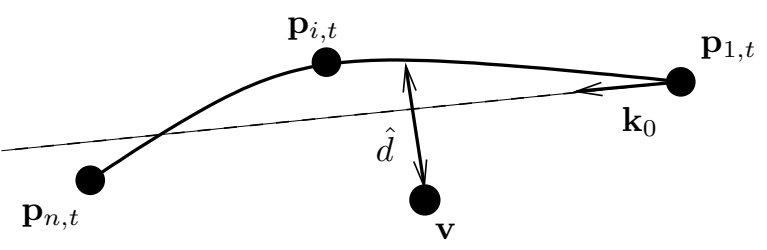

Fig. 1. Axis model and approximated point-to-curve distance. 
Once the axis of the needle is found, we determine the position of the tip along this axis by looking for an important intensity drop. As the appearance of the needle is not always contiguous, we discard small intensity gaps.

The major improvement on the localization stability is obtained by filtering the measurements of the detection algorithm using a Kalman filter, as described in section II-C.

\section{CONTROL SCHEME}

We propose to use the information provided by the localization to guide the ultrasound probe and follow the needle during the insertion. The ultrasound probe is mounted on a robotic arm with six degrees of freedom (DOF). The goal of the robotic task is to automatically maintain the needle visible, with its tip centered horizontally in the volume. Furthermore, we also propose to keep automatically the needle axis aligned with the longitudinal axis of the probe, in order to improve its visibility. To do so, we control 3 DOF by visual servoing:

- the two horizontal translations ( $X$ and $Z$ axes in Fig. 2) to keep the needle tip in the center of the image,

- and the rotation around $Y$ axis to align the needle with the $X$ axis.

The remaing translation, along the $Y$ axis (corresponding to the depth direction with respect to the patient surface), is controlled using force feedback to maintain a constant pressure on the body. We implemented the force control scheme presented in our previous work [8].

The visual servoing control scheme [23] consists in minimizing the error $\mathbf{e}(t)=\mathbf{s}(t)-\mathbf{s}^{*}$ between a set $\mathbf{s}(t)$ of observed visual features and a desired configuration $\mathbf{s}^{*}$. The visual features we use are the horizontal position $\left(X_{\mathrm{tip}}, Z_{\mathrm{tip}}\right)$ of the needle tip expressed in a Cartesian frame $\{X, Y, Z\}$ attached to the probe and the angle $\Theta$ between the principal direction $\mathbf{k}_{0}$ of the needle and the probe frame $X$ axis:

$$
\mathbf{s}=\left(X_{\mathrm{tip}}, Z_{\mathrm{tip}}, \Theta\right)
$$

The visibility of the needle is insured by fixing the desired features to the coordinates values $X_{C}$ and $Z_{C}$ of the volume center and a null angle:

$$
\mathbf{s}^{*}=\left(X_{C}, Z_{C}, 0\right)
$$

To obtain an exponential decrease of the visual error e we use the following classical control law [23]:

$$
\mathbf{v}_{\mathbf{c}}=-\lambda \widehat{\mathbf{L}_{\mathbf{s}}^{+}} \mathbf{e}
$$

where $\mathbf{v}_{\mathbf{c}}$ is the control velocity vector applied to the probe, $\lambda$ is a positive gain and $\widehat{\mathbf{L}_{\mathbf{S}}^{+}}$is the pseudo-inverse of an approximation of the interaction matrix $\mathbf{L}_{\mathbf{s}}$ that relates the variation of the feature $\mathbf{s}$ to the velocity $\mathbf{v}_{\mathbf{c}}$ :

$$
\dot{\mathbf{s}}=\mathbf{L}_{\mathbf{s}} \mathbf{v}_{\mathbf{c}}
$$

As we control 3 DOF by visual servoing, the velocity corresponds to $\mathbf{v}_{\mathbf{c}}=\left(v_{x}, v_{z}, \omega_{y}\right)$ where $v_{x}, v_{z}$ are the translational velocities along the $X$ and $Z$ axes of the probe frame and $\omega_{y}$ is the angular velocity around the $Y$ axis. The
TABLE I

IMAGING PARAMETERS

\begin{tabular}{c|c}
\hline Parameter & Value \\
\hline Gain & $42 \%$ \\
Depth & $9 \mathrm{~cm}$ \\
Sector & $75 \%$ \\
Tx-frequence & $3.3 \mathrm{MHz}$ \\
Focus depth & $2.5 \mathrm{~cm}$ \\
Degrees/Frame & 1.463
\end{tabular}

$3 \times 3$ interaction matrix related to the visual features $\mathbf{s}$ is given by :

$$
\mathbf{L}_{\mathbf{s}}=\left[\begin{array}{ccc}
-1 & 0 & -Z_{\text {tip }} \\
0 & -1 & X_{\text {tip }} \\
0 & 0 & 1
\end{array}\right]
$$

The visual servoing and the force control are managed separately by two different threads. As our servoing scheme only applies motion in a plane defined by $X$ and $Z$ axes, the two controls are perfectly decoupled.

\section{RESUlTS}

\section{A. Experimental setup}

For the acquisition of the volumes we use a SonixTOUCH Research US scanner (Ultrasonix Medical Corporation, Canada) and a motorized US 3D transducer (Model: 4DC7-3/40, Ultrasonix Medical Corporation, Canada). The imaging parameters of the transducer are summarized in Table I. The volumes are reconstructed from the raw data using the 3D scan conversion method described in [24], in order to compensate the deformations due to the motor sweeping. This 3D scan conversion is implemented on GPU using the CUDA language.

The probe is mounted on the end effector of a 6 DOF robot arm (Adept Viper s850, Adept Technology, Inc., USA). The scan conversion and the image processing is performed on a personal computer (Intel Xeon X5660 CPU @ 12x2.80GHz, NVIDIA GeForce GTX 480, 15.7GiB of random access memory), which receives the images from the US scanner and sends the commands to the robot. For the experiments we use a home-made agar phantom in which we insert manually a beveled needle. The phantom contains some imperfections that appear in the volume with intensities comparable to the needle intensity. In order to guide the operator during the insertion, we display two orthogonal views containing the needle axis (Fig. 3). The complete experimental setup is represented in Fig. 2.

\section{B. Localization}

We first validated our localization algorithm separately from the control algorithm, maintaining the probe still during the insertion. Fig. 4 shows the evolution of the tip position during the experiment when we insert (volume index intervals $0-200$ and 375-460) and take out (volume index intervals 200-375 and 460-660) the needle.

The experiment was repeated for different settings of the transducer, and the processing time of our algorithm was always shorter than the acquisition period, allowing real-time applications. 


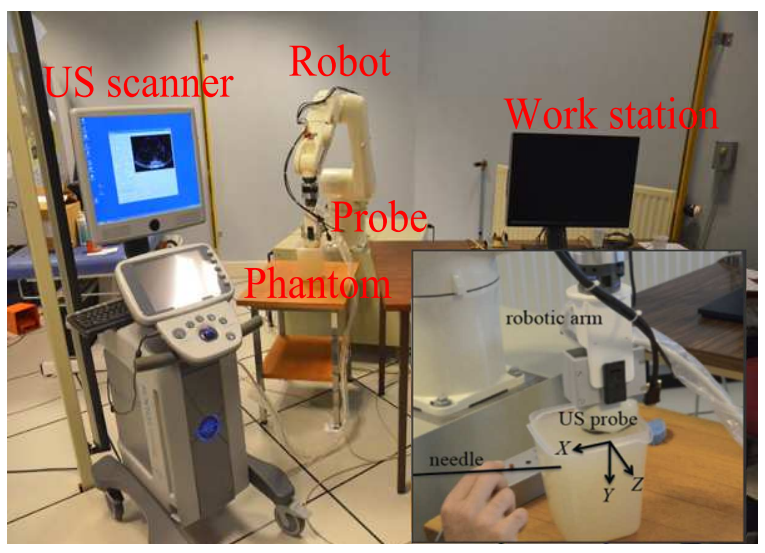

Fig. 2. Global view of the experimental setup: The US scanner, the robot holding the probe, the phantom and the work station performing the processes.

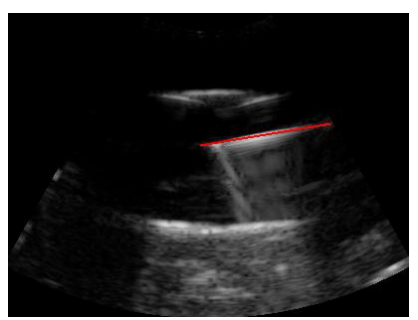

(a)

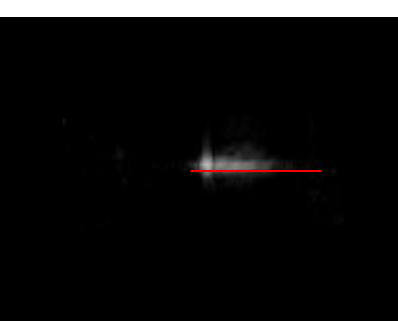

(b)
Fig. 3. The two orthogonal view planes. The estimated needle position is displayed in red.

\section{Control}

The automatic needle tracking by visual servoing was successfully tested in experiments on home-made agar phantoms, with various insertion directions. Fig. 5(a) shows the evolution of the feature error when the insertion axis was initially widely misaligned (by $40^{\circ}$ ) with the probe longitudinal axis. As expected, the feature error converges toward zero thanks to the visual servoing and the tip of the needle (Fig. 5(b)) is brought close to the coordinates $\left(X_{C}=13 \mathrm{~cm}, Z_{C}=6 \mathrm{~cm}\right)$ corresponding to the center of the volume. During the experiment the $Y$ coordinate of the tip is maintained quasi constant thanks to the force control scheme that insures a constant contact force of $2 \mathrm{~N}$ between the probe and the phantom. The displacement that was performed by the probe during the task is depicted in Fig. 5(c). Note that only the estimated feature error is represented, as we don't have access to the ground truth during manual insertion. When the operator stops to move the needle, the system maintains a mean feature error of $1.10 \mathrm{~mm}$ for the $X$-axis (which was the main direction of insertion), $0.23 \mathrm{~mm}$ for the $Z$-axis, and $0.97^{\circ}$ for the rotation $\Theta$.

To demonstrate the robustness of our tracking algorithm, we also performed experiments where the phantom was manually moved during the needle insertion (see the attached video). The results are presented in Fig. 6. Even with large and abrupt movements of the phantom that generate picks in the evolution of the tip position (translations of up to $4 \mathrm{~cm}$

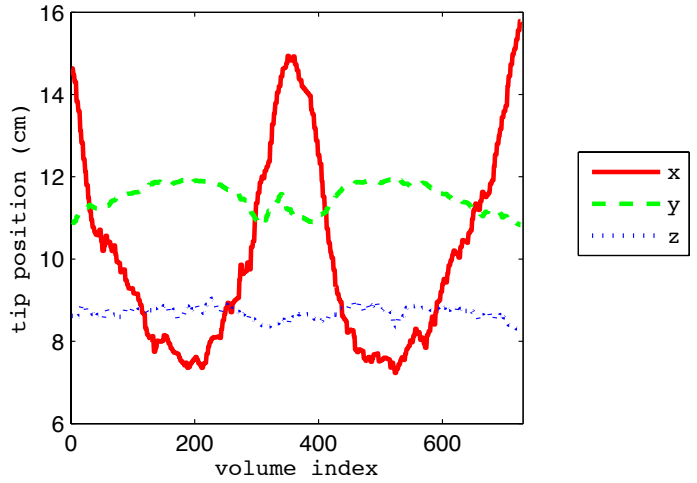

Fig. 4. Estimation of the tip position with a static probe. The volumes are acquired at a rate of 5.7 vols/s (1 volume index corresponds to $0.175 \mathrm{~s}$ ).

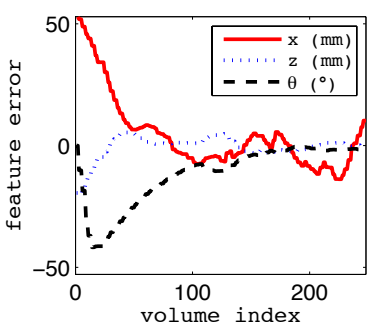

(a) Feature error.

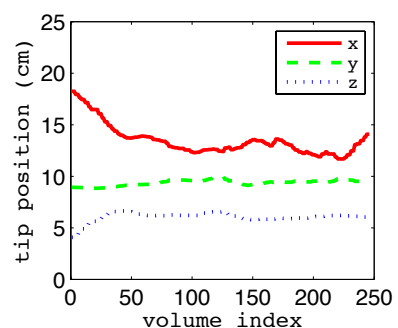

(b) Tip position.

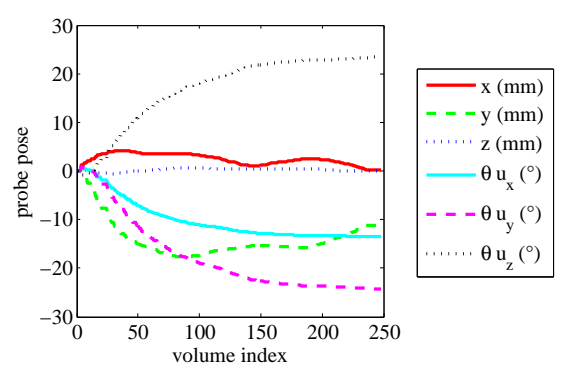

(c) Probe pose. The rotation is parameterized using the axis-angle $(\theta \mathbf{u})$ representation.

Fig. 5. Automatic needle tracking: Manual insertion with an initial angle of approximately $40^{\circ}$ between the needle axis and the longitudinal probe axis. The volumes are acquired at a rate of 3.3 vols/s (1 volume index corresponds to $0.303 \mathrm{~s}$ ).

along the $X$-axis, $1 \mathrm{~cm}$ along the $Y$-axis, and rotations of up to $20^{\circ}$ ), our system managed to stabilize itself and the feature error converged to zero in less than a few seconds (Fig. 6(a))

This robust behavior is very promising for real medical applications, where respiratory and other body motions disturb the acquisition.

\section{CONCLUSiON}

We designed an algorithm capable of detecting a needle inserted manually in a 3D US volume from an arbitrary point, and robustly tracking this needle in real-time. The next step to confirm the robustness of our method would be to perform experiments on real tissues. We also demonstrated the possi- 


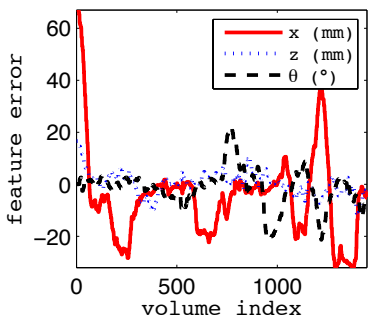

(a) Feature error.

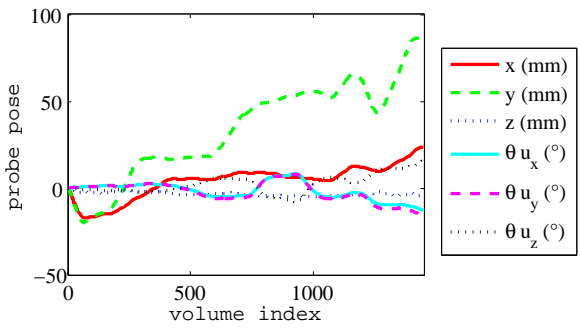

(c) Probe pose. The rotation is parameterized using the axis-angle $(\theta \mathbf{u})$ representation.

Fig. 6. Automatic needle tracking: Manual insertion in a moving phantom. The volumes are acquired at a rate of 3.3 vols/s (1 volume index corresponds to $0.303 \mathrm{~s}$ )

bility to guide the ultrasound probe to keep the needle visible and aligned, using visual servoing. Such a system could assist an operator during manual insertions, which are currently performed under free-hand US monitoring. In addition, this method can be combined in future work to a needle steering robotic system [18], [25], [26] to precisely guide the needle toward a target while optimizing its visibility.

\section{REFERENCES}

[1] G. A. Chapman, D. Johnson, and A. R. Bodenham, "Visualisation of needle position using ultrasonography," Anaesthesia, vol. 61, no. 2, pp. 148-158, 2006.

[2] S. Cheung and R. Rohling, "Enhancement of needle visibility in ultrasound-guided percutaneous procedures," Ultrasound in Medicine and Biology, vol. 30, no. 5, pp. 617-624, 2004.

[3] J.-S. Hong, T. Dohi, M. Hasizume, K. Konishi, and N. Hata, "A motion adaptable needle placement instrument based on tumor specific ultrasonic image segmentation," in International Conference on Medical Image Computing and Computer-Assisted Intervention (MICCAI'02), 2002, pp. 122-129.

[4] Z. Wei, M. Ding, D. Downey, and A. Fenster, "3d trus guided robot assisted prostate brachytherapy," in International Conference on Medical Image Computing and Computer-Assisted Intervention (MICCAI'05), 2005, pp. 17-24.

[5] P. Novotny, J. Stoll, P. Dupont, and R. Howe, "Real-time visual servoing of a robot using three-dimensional ultrasound," in IEEE International Conference on Robotics and Automation (ICRA'07), Apr. 2007, pp. 2655-2660.

[6] S. Salcudean, W. H. Zhu, P. Abolmaesumi, S. Bachmann, and P. D. Lawrence, "A robot system for medical ultrasound," in 9th International Symposium of Robotics Research (ISRR'99), Snowbird, 1999, pp. 152-159.
[7] R. Mebarki, A. Krupa, and F. Chaumette, "2-d ultrasound probe complete guidance by visual servoing using image moments," Robotics, IEEE Transactions on, vol. 26, no. 2, pp. 296 -306, Apr. 2010.

[8] C. Nadeau and A. Krupa, "Intensity-based direct visual servoing of an ultrasound probe," in IEEE International Conference on Robotics and Automation (ICRA'11), May 2011, pp. 5677 -5682.

[9] M. Aboofazeli, P. Abolmaesumi, P. Mousavi, and G. Fichtinger, "A new scheme for curved needle segmentation in three-dimensional ultrasound images," in IEEE International Symposium on Biomedical Imaging: From Nano to Macro (ISBI'09), July 2009, pp. 1067-1070.

[10] H. Zhou, W. Qiu, M. Ding, and S. Zhang, "Automatic needle segmentation in 3D ultrasound images using 3D improved Hough transform," in Society of Photo-Optical Instrumentation Engineers (SPIE) Conference Series, ser. Society of Photo-Optical Instrumentation Engineers (SPIE) Conference Series, vol. 6918, Apr. 2008.

[11] W. Qiu, M. Ding, and M. Yuchi, "Needle segmentation using 3d quick randomized hough transform," in International Conference on Intelligent Networks and Intelligent Systems (ICINIS'08), Nov. 2008 , pp. $449-452$.

[12] M. Barva, J. Kybic, H. Hlavac, H. Liebgott, and C. Cachard, "P3a-3 comparison of methods for tool localization in biological tissue from 3d ultrasound data," in IEEE International Ultrasonics Symposium (IUS'06), Oct. 2006, pp. 1983-1986.

[13] M. Uherčík, J. Kybic, H. Liebgott, and C. Cachard, "Multi-resolution parallel integral projection for fast localization of a straight electrode in 3d ultrasound images," in IEEE International Symposium on Biomedical Imaging: From Nano to Macro (ISBI'08), May 2008, pp. 33-36.

[14] H. Neshat and R. Patel, "Real-time parametric curved needle segmentation in 3d ultrasound images," in IEEE RAS EMBS International Conference on Biomedical Robotics and Biomechatronics, Oct. 2008, pp. 670-675.

[15] M. Uherčík, H. Liebgott, J. Kybic, and C. Cachard, "Neelde localization methods in 3d ultrasound data," in International Congress on Ultrasonics, no. 11, Jan. 2009.

[16] Y. Zhao, H. Liebgott, and C. Cachard, "Tracking micro tool in a dynamic 3d ultrasound situation using kalman filter and ransac algorithm," in IEEE International Symposium on Biomedical Imaging (ISBI'12), May 2012, pp. 1076 -1079.

[17] M. Uherčík, J. Kybic, H. Liebgott, and C. Cachard, "Model fitting using ransac for surgical tool localization in 3-d ultrasound images," IEEE Trans. Biomed. Eng., vol. 57, no. 8, pp. 1907 -1916, Aug. 2010.

[18] Z. Neubach and M. Shoham, "Ultrasound-guided robot for flexible needle steering," IEEE Trans. Biomed. Eng., vol. 57, no. 4, pp. 799 -805 , Apr. 2010

[19] E. Weiszfeld and F. Plastria, "On the point for which the sum of the distances to $\mathrm{n}$ given points is minimum," Annals of Operations Research, vol. 167, pp. 7-41, 2009.

[20] G. Welch and G. Bishop, "An introduction to the kalman filter," 1995.

[21] M. Uherčík, J. Kybic, C. Cachard, and H. Liebgott, "Line filtering for detection of microtools in 3d ultrasound data," in IEEE International Ultrasonics Symposium (IUS'09), Sept. 2009, pp. 594-597.

[22] J. A. Nelder and R. Mead, "A simplex method for function minimization," The Computer Journal, vol. 7, no. 4, pp. 308-313, Jan. 1965.

[23] F. Chaumette and S. Hutchinson, "Visual servo control. i. basic approaches," IEEE Robot. Automat. Mag., vol. 13, no. 4, pp. 82 90, Dec. 2006.

[24] D. Lee and A. Krupa, "Intensity-based visual servoing for non-rigid motion compensation of soft tissue structures due to physiological motion using 4d ultrasound," in IEEE/RSJ International Conference on Intelligent Robots and Systems (IROS'11), Sept. 2011, pp. 2831 $-2836$.

[25] R. Webster, J. S. Kin, N. Cowan, G. Chirikjian, and A. Okamura, "Nonholonomic modeling of needle steering," The International Journal of Robotics Research, vol. 25, pp. 509-525, May 2006.

[26] R. Alterovitz, M. Branicky, and K. Goldberg, "Motion planning under uncertainty for image-guided medical needle steering," The International Journal of Robotics Research, vol. 27, pp. 1361-1374, 2008 